\title{
Effect of Farnesol on Planktonic and Biofilm Cells of Staphylococcus epidermidis
}

\author{
Fernanda I. A. Gomes · Pilar Teixeira • \\ Joana Azeredo $\cdot$ Rosário Oliveira
}

Received: 11 December 2008/Accepted: 27 March 2009/Published online: 14 April 2009

(C) Springer Science+Business Media, LLC 2009

\begin{abstract}
Staphylococcus epidermidis is now amongst the most important pathogenic agents responsible for bloodstream nosocomial infections and for biofilm formation on indwelling medical devices. Its increasing resistance to common antibiotics is a challenge for the development of new antimicrobial agents. Accordingly, the goal of this study was to evaluate the effect of farnesol, a natural sesquiterpenoid, on Staphylococcus epidermidis planktonic and biofilm cells. Farnesol displayed a significant inhibitory effect on planktonic cells. Small concentrations $(100 \mu \mathrm{M})$ were sufficient to exhibit antibacterial effect on these cells. In biofilm cells the effect of farnesol was not so pronounced and it seems to be strongly dependent on the cells metabolic activity and amount of matrix. Interestingly, the effect of farnesol at $200 \mu \mathrm{M}$ was similar to the effect of vancomycin at peak serum concentration either in planktonic or biofilm cells. Overall, the results indicate a potential antibacterial effect of farnesol against $S$. epidermidis, and therefore the possible action of this molecule on the prevention of $S$. epidermidis related infections.
\end{abstract}

\section{Introduction}

Staphylococcus epidermidis and related coagulase-negative staphylococci (CoNS) are now well established as major nosocomial pathogens associated with infections of

F. I. A. Gomes $\cdot$ P. Teixeira $\cdot$ J. Azeredo $\cdot$ R. Oliveira $(\square)$ IBB-Institute for Biotechnology and Bioengineering, Centre of Biological Engineering, University of Minho, Campus de Gualtar, Braga 4710-057, Portugal

e-mail: roliveira@deb.uminho.pt indwelling medical devices $[2,3,15]$. This bacterium has become the primary cause of nosocomial bloodstream infections, also of the eye, ear, nose, and throat as well as cardiovascular infections [15]. This is related, in part, to the organism's ability to adhere to surfaces and form biofilms $[2,5,10,16]$. In fact, the formation of biofilms has been considered the main virulence mechanism of $S$. epidermidis $[10,15]$, and is the main cause of chronic infections [5]. Biofilm formation requires the bacterial attachment to solid surfaces, the development of bacterial multilayers and their enclosing in a large exopolymeric matrix [10]. This structure impairs the action of phagocytic cells from the immune system and of antimicrobial agents $[5,10,16]$, and releases planktonic cells from the outer layers, allowing the persistence of bacterial infections [10]. Biofilms are notoriously difficult to eradicate and are a source of many recalcitrant infections [16]. However, a more important consequence of biofilm formation, with profound clinical implications is the markedly enhanced resistance to antimicrobial agents by biofilm-associated microorganisms, which are estimated to be 50-500 times more resistant than their planktonic counterparts [6]. The ability of biofilm-embedded cells to resist to antimicrobial agents points to the importance of a continuous search for novel agents that are effective against bacteria in this mode of growth or that can work in synergy with the currently available myriad of antimicrobial agents [6]. Several studies have been assessing the antimicrobial potential of natural terpene alcohols. Specifically, farnesol has been reported to have antibacterial properties. For instance, in Streptococcus mutans, brief exposure to farnesol affected growth and metabolism by disrupting the bacterial membrane, as well as the accumulation and polysaccharide content of biofilms of the streptococci [6]. In Staphylococcus aureus, farnesol was shown to inhibit biofilm 
formation and compromise cell membrane integrity [6]. Inoue et al. [4] also demonstrated that farnesol have antibacterial activity against $S$. aureus. The results of these authors suggested that farnesol might act on cell membranes, where the damage to those membranes might be one of the major modes of action of this terpene alcohol [4].

Accordingly the goal of this study was to investigate the effect of farnesol on planktonic and biofilm cells of $S$. epidermidis.

\section{Materials and Methods}

\section{Bacterial Strains and Growth Conditions}

Four S. epidermidis strains were used in this study: two good biofilm-producing (1457 and 9142) and the respective mutants nonbiofilm-producing (1457-M10 and 9142-M10). These strains are clinical isolates and were stored at or below $-120^{\circ} \mathrm{C}$ in a liquid-nitrogen freezer and used as needed. All strains were provided by Dr. G. B. Pier, Channing Laboratory, Department of Medicine, Brigham and Women's Hospital, Harvard Medical School, Boston, USA. Tryptic Soy Broth (TSB) and Tryptic Soy Agar (TSA) were prepared according to the manufacturer's instructions. All strains were inoculated into $15 \mathrm{ml}$ of TSB from TSA plates not older than 2 days and grown for 24 $( \pm 2) \mathrm{h}$ at $37^{\circ} \mathrm{C}$ in an orbital shaker at $130 \mathrm{rpm}$. Cells were harvested by centrifugation (for $5 \mathrm{~min}$ at $9500 \mathrm{~g}$ and $4^{\circ} \mathrm{C}$ ), and resuspended in TSB adjusted to an optical density $(640 \mathrm{~nm})$ equivalent to $1 \times 10^{6}$ cells $\mathrm{ml}^{-1}$ and then used in the subsequent assays. Each stock solution of farnesol was prepared in methanol. It was confirmed that methanol, at the concentration used, had no effect on the growth of the S. epidermidis strains studied.

\section{Effect of Farnesol on Planktonic Cells}

\section{Farnesol Susceptibility of Planktonic Cells Assessed by Alamar Blue Assay}

Viability assays were performed in six well tissue-culture plates (Sarstedt, Newton, NC, USA) containing $4 \mathrm{ml}$ of a $S$. epidermidis cell suspension $\left(1 \times 10^{6}\right.$ cells ml $\left.{ }^{-1}\right)$ in the presence of farnesol $(0,30,100,150,200,250$, and $300 \mu \mathrm{M} ; 0-66 \mu \mathrm{g} \mathrm{ml}^{-1}$ ) (Sigma). The suspensions were incubated at $37^{\circ} \mathrm{C}$ and at $130 \mathrm{rpm}$. After 6 and $12 \mathrm{~h}$ of exposure to the different concentrations of farnesol, an aliquot of $200 \mu \mathrm{l}$ of $S$. epidermidis culture was removed from all experimental and control wells and $20 \mu \mathrm{l}(10 \% \mathrm{v} / \mathrm{v}$ of culture volume) of Alamar Blue (AB) (Biosource), an oxidation-reduction indicator, was added. Exposure of $A B$ to light was minimized throughout the experiment. The plates were shaken gently and incubated for $1 \mathrm{~h}$ at $37^{\circ} \mathrm{C}$. The growth was indicated by a change in colour from dark blue to pink. The tolerance threshold was defined as the lowest concentration of farnesol that prevented the development of a pink colour. This experiment was repeated twice, in triplicates.

\section{Farnesol Susceptibility of Planktonic Cells Assessed by CFU Plating}

Colony forming units (CFU) were obtained from the same wells used to obtain $\mathrm{AB}$ fluorescence as follows: $100 \mu \mathrm{l}$ of culture were removed from all experimental and control wells and the viable cells were determined by performing 10-fold serial dilutions in saline blanks and plated in TSA. Colonies were counted after $24 \mathrm{~h}$ at $37^{\circ} \mathrm{C}$. This experiment was repeated twice, in triplicates.

\section{Effect of Farnesol on Biofilm Cells}

\section{Farnesol Susceptibility of Biofilm Cells Assessed by Crystal} Violet and XTT Assays

In those assays only two $S$. epidermidis strains were used: S. epidermidis 1457 and S. epidermidis 9142, both good biofilm producers. Biofims were formed in 96 well tissue culture plates (Sarstedt, Newton, NC, USA) containing $200 \mu \mathrm{l}$ of a $S$. epidermidis cell suspension $\left(1 \times 10^{6}\right.$ cells $\mathrm{ml}^{-1}$ ) in TSB supplemented with $0.25 \%$ of glucose per well to promote biofilm formation. Plates were incubated at $37^{\circ} \mathrm{C}$ with orbital shaking at $130 \mathrm{rpm}$ for $24 \mathrm{~h}$. At the end, planktonic cells were removed carefully, and the biofilm was washed twice with $200 \mu \mathrm{l}$ of ultrapure water. The biofilms were incubated in fresh nutrient medium containing farnesol $(0,30,100,200$, and $300 \mu \mathrm{M})($ Sigma) and CV and XTT assays were performed at time 4, 8, and $24 \mathrm{~h}$. A similar assay was carried out to assess the effect of vancomycin at peak serum concentration ( $\mathrm{PS}=40 \mathrm{mg} \mathrm{l}^{-1}$ ) on $24 \mathrm{~h}$ biofilm cells. The aim of this assay was to compare the effect of farnesol with the effect of the most common "last resort" antibiotic used to control nosocomial infections. Crystal violet was used as indicator of total biofilm biomass. After exposure to farnesol and vancomycin, biofilms were washed with $200 \mu \mathrm{l}$ of $0.9 \% \mathrm{NaCl}$, then $250 \mu \mathrm{l}$ of methanol was added and allowed to act for $15 \mathrm{~min}$. Afterwards, methanol was removed and crystal violet was added $(5 \mathrm{~min})$. The wells were washed with water and finally, acetic acid 33\% (v/v) was added. The absorbance was measured at $570 \mathrm{~nm}$.

Another colorimetric method based on the reduction of XTT (\{2,3-bis(2-methoxy-4-nitro-5-sulfophenyl)-5-[(phenylamino)carbonyl]-2H-tetrazolium hydroxide $\}$ ) was 
applied to determine farnesol and vancomycin susceptibility (XTT is converted to a coloured formazan salt in the presence of metabolic activity) [8]. After exposure to antimicrobial agents, biofilms were washed with $200 \mu \mathrm{l}$ of $0.9 \% \mathrm{NaCl}$, then $250 \mu \mathrm{l}$ of a solution containing $200 \mathrm{mg} \mathrm{l}^{-1}$ of XTT and $20 \mathrm{mg} \mathrm{l}^{-1}$ of PMS (phenazine methosulphate) (Sigma) was added to each well. The microtiter plates were incubated for $3 \mathrm{~h}$ at $37^{\circ} \mathrm{C}$ in the dark. The absorbance was measured at $490 \mathrm{~nm}$.

Controls were biofilms not exposed to any antimicrobial agent tested. All experiments were carried out in triplicate and repeated three times.

\section{Statistical Analysis}

The data from all assays were compared using one-way analysis of variance (ANOVA) by applying Tukey's and Bonferroni tests with all calculations carried out using SPSS software (Statistical Package for the Social Sciences). Differences achieving a confidence level of $95 \%$ were considered significant.

\section{Results and Discussion}

Four S. epidermidis strains were selected for this study: strain 1457 is a producer of the polysaccharide intercellular adhesin (PIA), the major surface polysaccharide (poly- $N$ acetyl glucosamine or simply PNAG) promoting coagulase-negative staphylococci intercellular adherence and biofilm formation, and its isogenic biofilm-negative mutant icaA::Tn917 transductant 1457-M10 [11]; S. epidermidis 9142 another well-known producer of PIA and S. epidermidis $9142-\mathrm{M} 10$ an isogenic strain to 9142 , containing a transposon inserted into the ica locus which encodes the biosynthetic enzymes for producing PIA/PNAG and thus does not produce this molecule [2]. The growth inhibition effect of farnesol on planktonic cells of S. epidermidis was assessed using a rapid colorimetric assay that measures cellular metabolic activity and is based on the reduction of Alamar Blue. The viability assays showed, for all S. epidermidis strains used, that incubation of planktonic cells in the presence of $100 \mu \mathrm{M}\left(22 \mu \mathrm{g} \mathrm{ml}^{-1}\right)$ farnesol resulted in no change in colour from blue to pink, indicating that this concentration inhibited oxidation-reduction reactions in the suspensions (data not shown). These data indicated that low concentrations of farnesol were sufficient to exhibit antibacterial effect, as was demonstrated by viability assays. For Staphylococcus aureus this was observed in the presence of $200 \mu \mathrm{M}\left(44 \mu \mathrm{g} \mathrm{ml}^{-1}\right)$ farnesol [6]. This inhibitory effect can be due to the hydrophobic nature of farnesol that favours its accumulation in the membrane, possibly causing membrane disruption [6]. In fact, exposure to terpene alcohols has been recently shown to affect the cell membranes of $S$. aureus, Escherichia coli and Listeria monocytogenes, resulting in leakage of $\mathrm{K}^{+}$ions from cells [6].

Figure 1 depicts the effect of different farnesol concentrations on planktonic cells of the four strains studied. Generally, there was a steep decrease in CFUs at $100 \mu \mathrm{M}$ of farnesol $(P<0.05)$, levelling off for higher concentrations. A farnesol concentration of $100 \mu \mathrm{M}$ seems to be sufficient to promote a very significant reduction in $S$. epidermidis planktonic cells viability. It should be stressed that for concentrations above $100 \mu \mathrm{M}$, the cellular viability remains almost the same independently of farnesol concentration and exposure time. In fact, it seems that there is a threshold of farnesol uptake acting as limiting factor rather than farnesol concentration. Besides, bacterial populations produce persister cells that neither grow nor die in the presence of microbicidal agents. Persisters are largely responsible for high levels of biofilm tolerance to antimicrobials [7]. This was also confirmed in experiments with E. coli, where a small sub-population of cells remained alive irrespective of the concentration of the antibiotic (persisters) [9].
A

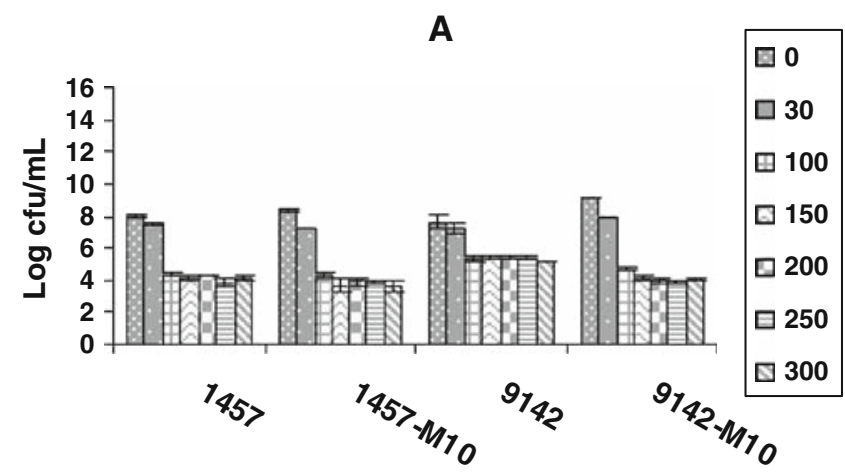

B

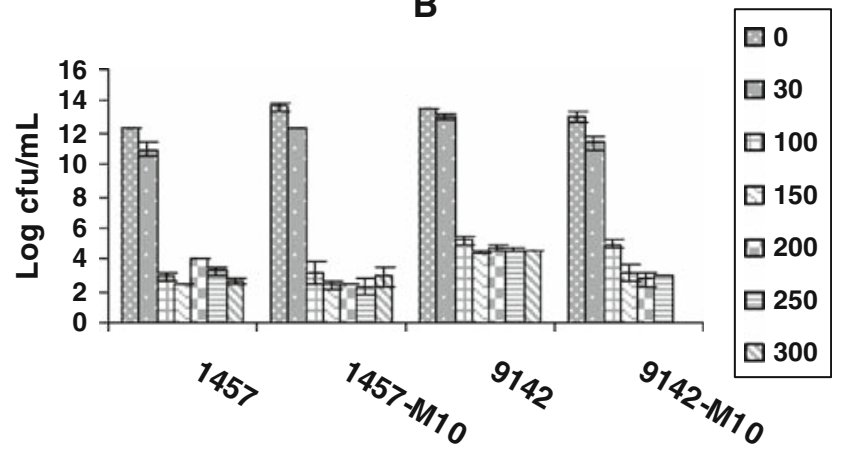

Fig. 1 Effect of farnesol $(0-300 \mu \mathrm{M})$ on S. epidermidis planktonic cells assessed by CFU enumeration, after 6 (a) and $12 \mathrm{~h}$ (b) of exposure to farnesol. Bars represent standard deviation of the mean 
According to the other goal of this work, farnesol was added to $24 \mathrm{~h}$ biofilms of S. epidermidis 1457 and 9142 strains and biofilm biomass and activity were evaluated. These two strains were selected because they are both good biofilm producers. As could be expected, biofilm cells were much less sensitive to farnesol than their planktonic counterparts. Noticeably, strain 9142 biofilm was more sensitive to farnesol than the sessile cells of strain 1457 (Figs. 2 and 3). This corresponds to an inversion of the observed behaviour for planktonic cells (Fig. 1). Those facts prove that the biofilm structure along with the specific physiology of the sessile mode of life determine a response to antimicrobial agents, which cannot be extrapolated from tests performed with planktonic cells.

To have some comparison of the response of biofilm cells it was decided to assess the effectiveness of vancomycin, one of the most frequently used antibiotics to treat resistant nosocomial infections, which is primarily effective against coagulase negative Staphylococcus species [12]. Furthermore, both tested agents act at the cell wall level, vancomycin inhibits the biosynthesis of bacterial cell wall, while farnesol is considered to disrupt the normal barrier function of the cell membrane [1]. Interestingly, farnesol at a concentration higher than $200 \mu \mathrm{M}$ displayed the same or higher effectiveness of vancomycin at peak serum concentration (Figs. 2 and 3). In fact, the response of the strains tested was very similar for both farnesol $(>200 \mu \mathrm{M})$ and vancomycin. Accordingly, the biofilm of strain 1457 showed higher tolerance to vancomycin than the biofilm of strain 9142. In a previous study, Sousa et al. [13] have shown that planktonic cells of strain 1457 displayed a higher glucose uptake, compared to 9142 planktonic cells. Conversely, in biofilm form the glucose uptake of strain 9142 was 3.5 times higher than that of sessile cells of strain 1457. This suggests that cell susceptibility increases with cell metabolic activity. Despite the effect of specific changes in cell physiology triggered by the sessile form of life, the higher amount of exopolysaccharides present in the biofilm matrix of strain 1457 [14], can also play a role in the increased tolerance to the antimicrobials tested. This is another point in favour of the virulence character of the matrix.

As conclusion, the results show the significant effect of farnesol in the reduction of the viability of planktonic cells of the four S. epidermidis strains assayed and less pronounced on mature biofilm cells. In fact, on planktonic cells, small concentrations of this sesquiterpenoid $(100 \mu \mathrm{M})$ are sufficient to exhibit antibacterial effect, as was shown by viability assays. Additionally, the loss of cellular viability and consequently the loss of biofilm formation ability (main virulence factor) induced by farnesol suggest a potential use of this molecule in the prevention of S. epidermidis infection.

It was once again demonstrated that the response of planktonic cells cannot be extrapolated for biofilm cells and this must be a driving force for the development of new
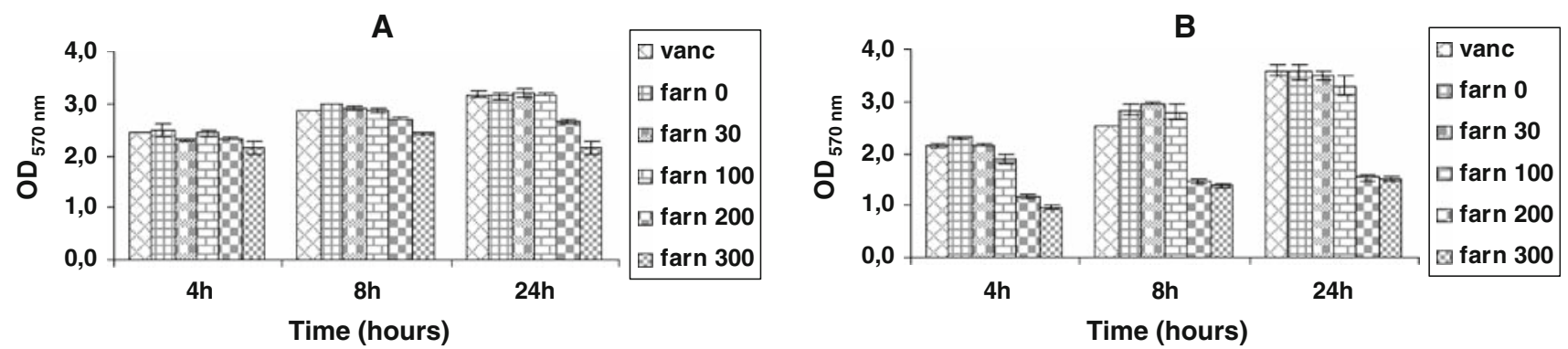

Fig. 2 Effect of farnesol (farn) $(0-300 \mu \mathrm{M})$ and vancomycin (vanc) $\left(40 \mathrm{mg} \mathrm{l}^{-1}\right)$ on biofilm cells of S. epidermidis 1457 (a) and 9142 (b), expressed as CV absorbance (total biofilm biomass)
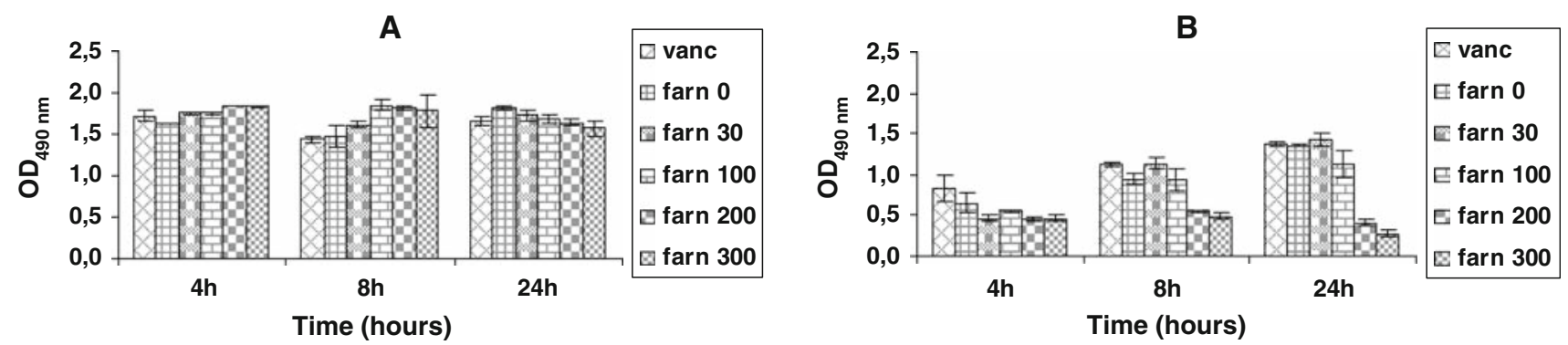

Fig. 3 Effect of farnesol (farn) $(0-300 \mu \mathrm{M})$ and vancomycin (vanc) $\left(40 \mathrm{mg} \mathrm{l}^{-1}\right)$ on biofilm cells of S. epidermidis 1457 (a) and 9142 (b), expressed as XTT absorbance (cellular activity) 
methodologies to assess sessile cells susceptibility to antimicrobial agents.

Acknowledgements Fernanda Gomes and Pilar Teixeira fully acknowledge the financial support of Fundação para a Ciência e Tecnologia (FCT) through the grants SFRH/BD/32126/2006 and SFRH/BPD/26803/2006, respectively.

\section{References}

1. Brehm-Stecher BF, Johnson EA (2003) Sensitization of Staphylococcus aureus and Escherichia coli to antibiotics by the sesquiterpenoids nerolidol, farnesol, bisabolol, and apritone. Antimicrobial Agents Chemother 47:3357-3360

2. Cerca N, Pier GB, Oliveira R et al (2004) Comparative evaluation of coagulase-negative staphylococci (CoNS) adherence to acrylic by a static method and a parallel-plate flow dynamic method. Res Microbiol 155:755-760

3. Cerca N, Martins S, Cerca F et al (2005) Comparative assessment of antibiotic susceptibility of coagulase-negative staphylococci in biofilm versus planktonic culture as assessed by bacterial enumeration or rapid XTT colorimetry. J Antimicrob Chemother 56:331-336

4. Inoue Y, Shiraishi A, Hada T et al (2004) The antibacterial effects of terpene alcohols on Staphylococcus aureus and their mode of action. FEMS Microbiol Lett 237:325-331

5. Izano EA, Sadovskaya I, Vinogradov E et al (2007) Poly- $N$ acetylglucosamine mediates biofilm formation and antibiotic resistance in Actinobacillus pleuropneumoniae. Microb Pathog 43:1-9
6. Jabra-Rizk MA, Meiller TF, James CE et al (2006) Effect of farnesol on Staphylococcus aureus biofilm formation and antimicrobial susceptibility. Antimicrob Agents Chemother 50:1463-1469

7. Keren I, Kaldalu N, Spoering A et al (2003) Persister cells and tolerance to antimicrobials. FEMS Microbiol Lett 230:13-18

8. Kuhn DR, Balkis M, Chandra J et al (2003) Uses and limitations of the XTT assay in studies of Candida growth and metabolism. J Clin Microbiol 41:506-508

9. Lewis K (2007) Persister cells, dormancy and infectious disease. Nat Rev Microbiol 5:48-56

10. Oliveira M, Nunes SF, Carneiro C et al (2007) Time course of biofilm formation by Staphylococcus aureus and Staphylococcus epidermidis mastitis isolates. Vet Microbiol 124:187-191

11. Rupp ME, Ulphani JS, Fey PD et al (1999) Characterization of the importance of polysaccharide intercellular adhesin/hemagglutinin of Staphylococcus epidermidis in the pathogenesis of biomaterial-based infection in a mouse foreign body infection model. Infect Immun 67:2627-2632

12. Rybak MJ (2006) The pharmacokinetic and pharmacodynamic properties of vancomycin. Clin Infect Dis 42:S35-S39

13. Sousa C, Henriques M, Azeredo J et al (2008) Staphylococcus epidermidis glucose uptake in biofilm versus planktonic cells. World J Microbiol Biotechnol 24:423-426

14. Sousa C, Teixeira P, Oliveira R (2009) The role of extracellular polymers on Staphylococcus epidermidis biofilm biomass and metabolic activity. J Basic Microbiol 49:1-8

15. Vuong C, Gerke C, Somerville GA et al (2003) Quorum-sensing control of biofilm factors in Staphylococcus epidermidis. J Infect Dis 188:706-718

16. Wang C, Li M, Dong D et al (2007) Role of ClpP in biofilm formation and virulence of Staphylococcus epidermidis. Microb Infect 9:1376-1383 